SUPERCLUSTERING: THEORY VERSUS OBSERVATIONS

\author{
Jaan Einasto, Maret Einasto and Enn Saar \\ Tartu Astrophysical Observatory, Toravere, Estonia, USSR \\ Bernard J. T. Jones and Vicent J. Martinez \\ NORDITA, Copenhagen, Denmark
}

\begin{abstract}
The spatial distribution of galaxies is compared with model distributions. It is demonstrated that giant and dwarf galaxies in the Local Supercluster occupy statistically identical regions. Various tests suggest that galaxy formation is biased since all unbiased model distributions are in conflict with observed distribution of galaxies. Multifractal analysis shows that a cold dark matter dominated universe with biased galaxy formation has a fairly constant fractal dimension over a broad range of scales. This contrasts with the observed distribution which does not show simple fractal features.
\end{abstract}

\title{
1 INTRODUCTION
}

Our understanding of the formation and evolution of the large scale structure in the Universe has a number of weak points: we have still no firm evidence on the nature of dark matter, very little is known on physical processes involved in galaxy formation, there is no clear understanding how to explain the presence of large voids and coherent motion of superclusters. In this report we shall discuss the large scale distribution of galaxies and compare observations with theoretical models.

Currently popular structure formation scenarios are based on either hot or cold dark matter (CDM) dominated universes. Observational predictions of these rivaling scenarios are different. Both scenarios have one difficulty in common: not all test particles can be identified with galaxies. Some particles remain in primordial form and do not participate in galaxy formation. The absence of galaxy formation in low density regions was emphasized already by Zeldovich and his collaborators (Zeldovich, Einasto and Shandarin 1982 and references therein). Now this phenomenon is called biased galaxy formation (Kaiser 1984). The biasing mechanism is physically related to processes involved in galaxy formation. Since our knowledge on these processes is still poor we must first study biasing from an empirical point of view by comparing the distribution of test particles in biased model samples with the distribution of galaxies.

J. Audouze et al. (eds.), Large Scale Structures of the Universe, 245-254. (c) 1988 by the IAU. 
A strong test of the rival scenarios is the spatial distribution of galaxies of different luminosity. In CDM dominated scenarios the distribution should depend on the luminosity. It is expected that giant galaxies populate preferentially high density regions but dwarf galaxies can be found also in a low density environment (Dekel and Silk 1986). In hot dark matter scenarios this problem has not been addressed specifically, but no prominent difference in spatial distribution is expected (Doroshkevich, Shandarin and Saar 1978).

To test the scenarios and, more important, to obtain a sensible description of the complex geometry of the large-scale structure, a number of authors (Mandelbrot 1982, Lachieze-Rey 1986) have proposed fractàl models of the galaxy distribution. It is not clear, however, of the observed distribution has fractal features or not.

In the following we give a review of our recent results concerning these problems. We have used conventional tests as well as some new ones - the distribution of nearest neighbors and the fractal and multifractal analysis. More detailed reports are in preparation (Saar et al. 1987, Einasto 1987, Jones et al. 1987).

\section{THE DISTRIBUTION OF BRIGHT AND FAINT GALAXIES}

In a recent study we have found that voids are essentially empty: they contain neither giant nor dwarf galaxies (Einasto et al. 1986a). Now we address a different problem: do giant and dwarf galaxies populate identical or various regions? In the second case galaxies of various magnitude could populate regions of differing mean density, say dwarfs forming low density halos around densely packed cores of giant galaxies in clusters, as observed in groups with one concentration center.

To get an answer to these questions Einasto (1987) compared the distribution of galaxy samples with a number of test samples. The sample Virgo A contains galaxies brighter than -17.5, the sample Virgo $B$ consists of galaxies with absolute magnitudes between $-\mathbf{1 5 . 0}$ and $\mathbf{- 1 7 . 5}$, the sample Virgo $C$ is the sum of Virgo $A$ and $B$. Absolute magnitudes correspond to Hubble constant $\mathrm{H}=100 \mathrm{~km} / \mathrm{s} / \mathrm{Mpc}$, and distances are given in units of $h^{-1} \mathrm{Mpc}$, where $h$ is the Hubble constant in units of 100 $\mathrm{km} / \mathrm{s} / \mathrm{Mpc}$. The absolute magnitude $\mathbf{- 1 7 . 5}$ corresponds to the apparent magnitude limit, $m=14.5$, of the CfA redshift survey at the far end of the samples. The samples have a cubic geometry, being approximately centered on the Virgo cluster. The supergalactic coordinates $\mathrm{X}_{0}, \mathrm{Y}_{0}, \mathrm{Z}_{0}$, of the center, the cube size $\mathrm{L}$, the number of galaxies, $N$, and the absolute magnitude limit, $M_{0}$, are given in Table 1.

The sample Virgo A is essentially complete, but the sample Virgo $B$ is incomplete in respect to dwarf galaxies. However, in the box under study it contains all dwarf spiral and irregular galaxies observed by Fisher and Tully (1981) in their radio survey which has a limiting redshift about $3200 \mathrm{~km} / \mathrm{s}$, thus being the most complete dwarf sample with measured redshifts currently available.

A pair of test galaxy samples was generated with statistically identical spatial distribution. For this purpose the combined sample Virgo $C$ was divided at random into two equal parts, $C 1$ and $C 2$. To find the sensitivity of the method galaxy samples with shifted spatial dis- 
tribution were generated by adding to $X, Y$ and $Z$ coordinates of all galaxies in the sample Virgo $B 200 \mathrm{~km} / \mathrm{s}$ in redshift space. Finally the sample Virgo $C$ was divided by cluster analysis into two parts, $C_{c l}$ and $C_{i}$, containing galaxies in high and low density environment, respectively. The neighborhood radius was chosen to get approximately equal number of galaxies in both parts.

Table 1. Data on observed samples

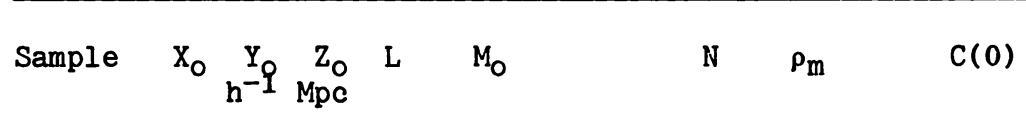

\begin{tabular}{|c|c|c|c|c|c|c|c|c|}
\hline 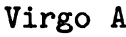 & 0 & 15 & 0 & 20 & -17.5 & 524 & 0.128 & 0.308 \\
\hline $\mathrm{B}$ & 0 & 15 & 0 & 20 & $-15.0-17.5$ & 488 & 0.119 & \\
\hline$C$ & 0 & 15 & 0 & 20 & -15.0 & 1012 & 0.247 & \\
\hline
\end{tabular}

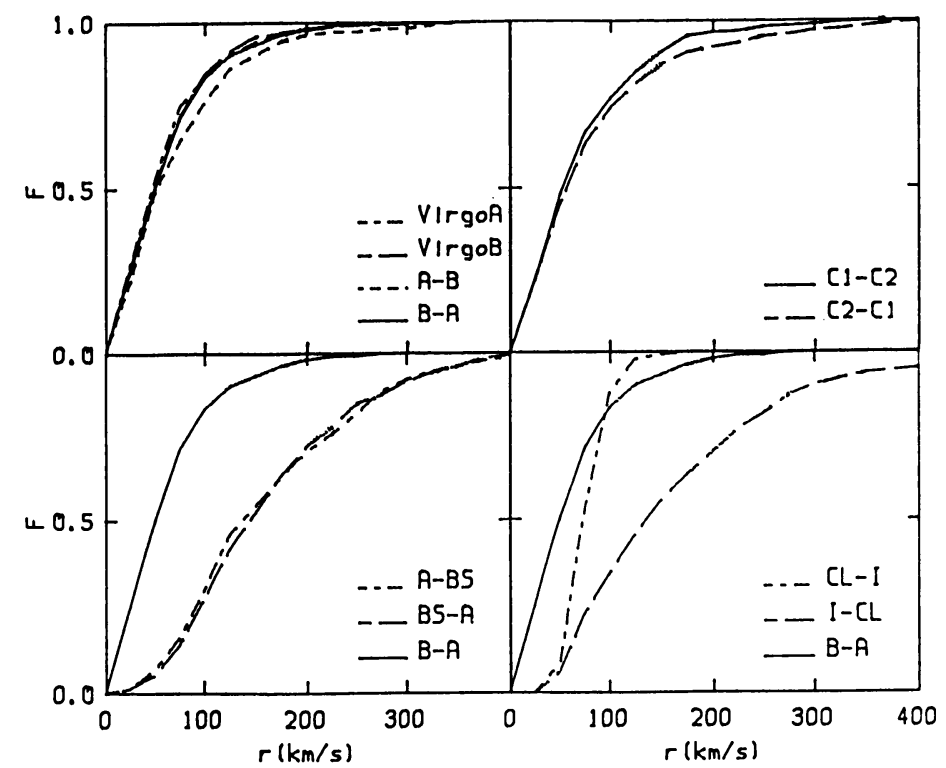

Fig. 1. The integral nearest neighbors distributions for samples Virgo A and B sample pairs A-B, B-A (panel a), sample pairs Virgo C1 and C2 (panel b), pairs Virgo $A$ and shifted Virgo $B$ (BS, panel $c$ ), and clustered and isolated galaxy sample pairs Virgo $C_{c l}$ and Virgo $C_{i}$ (panel d). For reference the last panel includes the distribution for the pair B-A.

After some trial it was found that the most sensitive statistic to compare the spatial location is the cross distribution of nearest neighbors for all samples and sample pairs: the distribution of nearest giants (Virgo A) for all dwarfs (Virgo B) and vice versa etc. The resul- 
ting distributions are plotted in Fig. 1. The number of galaxies in all samples is approximately equal, which makes the comparison of distributions easy (instead of normalized radii we can use absolute neighborhood radii as an argument of the nearest neighbor distribution).

The comparison of results demonstrates that the nearest neighbor distributions for bright and faint galaxies are practically identical and, moreover, coincide with the pairwise distributions A-B, B-A. The Kolmogorov test tells the same - all distributions coincide at a 99\% confidence level. This result tells us that the samples $A$ and $B$ cover the same region.

Additional evidence for this fact is given by panel $b$, which shows that by randomly dividing the whole sample (bright plus faint galaxies) we get the same results as for samples of different luminosity.

On the contrary, the spatial distributions of clustered and isolated galaxies are extremely different, as demonstrated by panel d. And, of coarse, the pair distributions for shifted samples differ strongly from those for the real samples. The last result shows that the nearest neighbor test is rather sensitive to even small differences in spatial distribution.

We come to the conclusion that bright and faint galaxies populate statistically identical regions in space. Presently we do not know whether the Virgo Supercluster is representative for the whole Universe or not. To check this point larger and deeper dwarf galaxy samples are needed.

\section{BIASED GALAXY FORMATION}

An important property of the observed distribution of galaxies is the absence of visible objects between galaxy filaments and superclusters. Numerical simulations represent the formation of a connected network of filaments. However, simulations also demonstrate the presence of a striking difference between theory and observations: in simulations there exists a more or less homogeneous population of non-clustered test particles, which has no counterpart in the observed distribution.

Analytical calculations suggest that it is impossible to evacuate voids between galaxy filaments completely, since gravitation, the only force responsible for the formation of large scale structure, works very slowly (Einasto, Joeveer and Saar 1979). Thus there must be primeval particles in voids, seen in numerical simulations as the population of field particles. This discrepancy between simulations and observations has a simple explanation: suppose that galaxies form only in a high-density environment, and in regions of low density matter remains in the primeval form (Zeldovich, Einasto and Shandarin 1982). To bring simulations into agreement with observations we have used two biasing schemes in the present study. In the first case the bias level $n$ is given by the threshold density in units of mean density at the start of model calculations corresponding to the end of the linear regime, and in the second case - at the present epoch.

We must stress that both of the biasing rules used are extremely simplified and have been chosen only by their present popularity among astronomers. The real goal of biasing is to transform the distribution 
of total density into distribution of galaxies, and thus a good biasing scheme must mimic the galaxy formation process as well as possible. So far no biasing scheme has been built on this principle, and, consequently, all our comparisons between simulations and observations stand in doubt. The problem of galaxy formation and physically justified biasing seems to be one of the most urgent project in cosmology.

At present we consider the results presented below as giving some hints to be taken into account in constructing future biasing prescriptions. Tables and Figures correspond to the first biasing case (both cases yield rather similar results).

The numerical simulation used (Gramann 1987) corresponds to a CDM dominated scenario with a positive cosmological constant. The input parameters of the model were adjusted to get $\Omega_{\lambda}=0.8$ and $\Omega_{\text {matter }}=0.2$ for the present epoch. The simulation was made with $64^{3}$ particles in a $64^{3}$ mesh. The data on model samples are given in Table 2, where samples are designated by $G R-n$, index $n>0$ denoting the bias level, and index 0 labeling unbiased samples with all test particles included.

Table 2. Data on model samples

\begin{tabular}{crrrrr}
\hline Sample & L & $\begin{array}{r}\text { Bias } \\
\text { level }\end{array}$ & $N$ & $\rho_{\mathrm{m}}$ & $\mathrm{N}(0)$ \\
\hline GR-0 & 40 & 0.0 & 262144 & 8.000 & 1.000 \\
1.0 & 40 & 1.0 & 174227 & 5.317 & 0.675 \\
1.5 & 40 & 1.5 & 115802 & 3.534 & 0.453 \\
2.0 & 40 & 2.0 & 75825 & 2.314 & 0.297 \\
2.5 & 40 & 2.5 & 50823 & 1.551 & 0.196 \\
3.0 & 40 & 3.0 & 35160 & 1.073 & 0.126 \\
3.5 & 40 & 3.5 & 25100 & 0.766 & 0.088 \\
4.0 & 40 & 4.0 & 18055 & 0.551 & 0.066
\end{tabular}

For both biasing rules we have varied the bias level and compared the distribution of test particles with the observed distribution of galaxies in the Virgo Supercluster. Several methods have been tried, and two of them have been found to be rather sensitive to the bias level, the filling factor test and the multiplicity distribution of particles.

3,1 Filling factor test. In this test we calculated the filling factor of model and observed samples for a wide interval of threshold densities which divide the space into the 'empty' and 'filled' regions. To do so we calculated a smooth density field for all samples using a cubic 'top hat' smoothing method as customary in numerical modeling of structure evolution (Hockney and Eastwood 1981). The smoothing length was taken as $1=1.25 \mathrm{~h}^{-1} \mathrm{Mpc}$.

The filling factor of model samples depends critically on the bias level. In unbiased samples the density is nonzero over the whole space, thus at zero threshold density practically the whole space is 'filled'. 
All biased samples contain really empty regions of zero density. The volume of these empty regions is the larger the higher is the bias level. The filling factor at zero threshold density, $C(0)$, decreases with growing bias level, reaching $C(0)=0.066$ for the bias level 4.0 (see Table 2). The real Universe also contains regions completely void of luminous galaxies, and the filling factor at zero threshold density level $C(0)=0.308$. This value determines the bias level which brings models into agreement with observations, to be close to $n=2$. The filling factor versus threshold density curves are presented in Fig. 2.

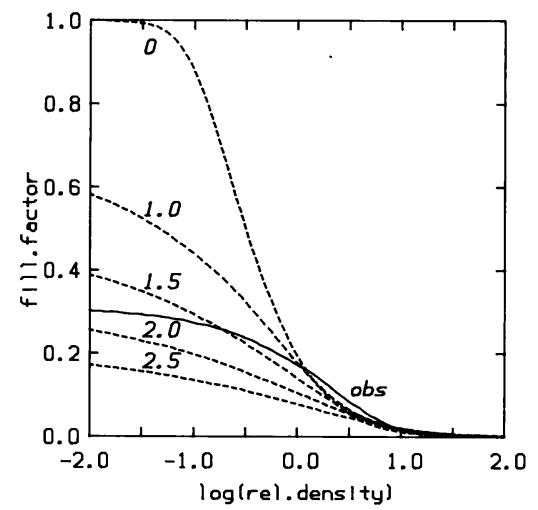

Fig. 2. Filling factor versus threshold density for model and observed samples. Model samples are labeled with the bias level parameter $n$, the value $n=0$ corresponding to unbiased samples.

3.2 Multiplicity test. We use the multiplicity function to determine the fraction of quasi-randomly distributed particles. To make calculations easier and the mean density of particles per unit volume, $\rho_{m}$, in model samples closer to the respective density in observed samples we have picked from the original sample $32^{3}$ random particles. From this sample we chose subsamples for different bias levels. Thereafter by cluster analysis the fraction of single (isolated) objects, and of systems of higher multiplicity has been found. The multiplicity function has been calculated for a series of neighborhood radil, and the results are presented in Fig. 3 .

As expected, the fraction of isolated galaxies is sensitive to the bias level. Unbiased samples have the largest fraction of isolated galaxies for a given neighborhood radius (radii are expressed in units of the Poisson radius, i.e. the radius of a sphere which contains in the mean one particle). With increasing bias level the fraction of isolated particles decreases, and relatively more particles are located in large systems. Best agreement with observations is achieved for a bias level $\mathrm{n}=1$. We see also that in case of systems of higher multiplicity the model curves fit observations rather poorly.

3.3 Discussion of bias level tests. The filling factor and multiplicity tests both demonstrate the need for biased galaxy formation. The bias level needed to bring models into agreement with observations is, how- 
ever, different. The mean density, $\rho_{m}$, of particles per cell of length 1 in model samples is much higher than in the observed one (see Tables 1 and 2). To clarify possible reasons for this discrepancy we have studied the density dependence of both tests by generating model subsamples with smaller number of particles.

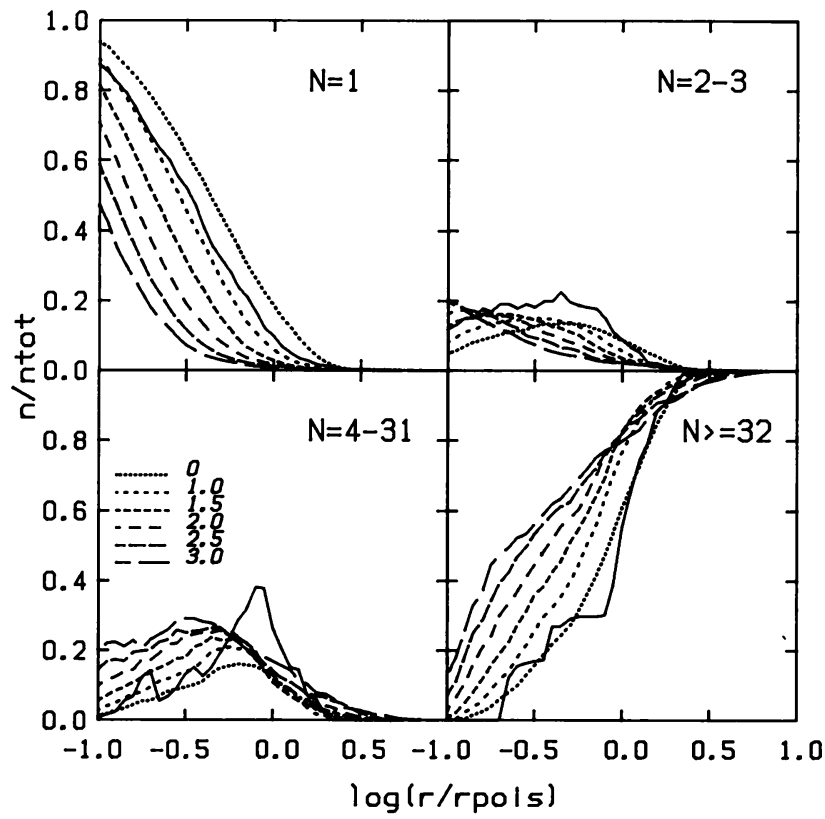

Fig. 3. The multiplicity function versus neighborhood radius for isolated particles and systems of multiplicity $2-3,4-31$, and 32 and higher for the observed and model samples. The observational results are given by solid line, and model curves by dashed lines marked by their bias level.

Surprisingly enough, we come to the conclusion that principal properties of the multiplicity distribution are fairly stable and almost independent on the number density of samples. A similar check indicates that filling factors are also practically identical for model samples with both high and low mean particle densities. Thus the reason for the discrepancy must lie elsewhere.

One possibility to explain the discrepancy may be the fact, that all test particles in model calculations left over after the biasing procedure have been identified with galaxies. This may not be the best biasing scheme. Actually galaxies can be formed by coalescing of a number of test particles, as suggested by White et al. (1986). If this is the case then we cannot use the multiplicity function test in the present form since isolated particles may not correspond to real galaxies.

Whatever bias level is the best, it must be the same for both giant and dwarf galaxies since the distribution of all galaxies is similar. 


\section{FRACTAL DESCRIPTION OF LARGE-SCALE STRUCTURE}

The complicated geometry of superclusters does not lend itself easily to quantitative description. More or less regular patterns of this geometry are often deformed by seemingly irregular features. One possible way to follow is the study of the topology (connectedness) of large scale structure (Gott, Melott and Dickinson 1986, Einasto et al. 1986b) and another one seems to be the use a fractal description of this geometry (Mandelbrot 1982).

In using the fractal description we are confronted with the following problem: is there any scale interval over which the geometry of superclusters can be considered approximately self-similar? To answer this question one needs to determine the fractal dimension $D$ of known samples of galaxies for different scales. We use here an analog of the capacity dimension

$$
D(1)=\frac{d(\ln N(1))}{d(\ln 1 / 1)}
$$

obtained by counting the sample volume with cubes of side 1 (the scale) and counting the number of cubes containing galaxies (N). This dimension corresponds to the exponent in the relation $M(r) \sim r^{D}$, usually used to calculate fractal dimensions, where $M$ is the mean number of galaxies in a sphere of radius $r$. The first tests of this kind have been done by Jones et al. (1987) and show that one can find a self-similarity (D is constant for a significant scale interval) only in case of numerical simulations (as before, the CDM scenario with cosmological term was used). The Coma sample exploited for the fractal dimension test is conical, has a limiting redshift $\mathrm{V}_{0}=8000 \mathrm{~km} / \mathrm{s}$, magnitude limit $\mathrm{M}_{0}=-20$, declination limit $\delta \geq 0^{\circ}$, galactic latitude limit $b \geq 40^{\circ}$, and contains 425 galaxies. As seen from Fig. 4, the fractal dimension of the real sample changes continuously. The Coma sample consists only of supergiant galaxies of low spatial density, thus it does not describe well the fine structure on scales $1 \leq 5 \mathrm{~h}^{-1}$ Mpc.

Another problem that must be answered is the possibility of the existence of a mixture of fractal sets in the data. This sort of geometry is predicted, e.g. by the classical adiabatic or 'pancake' theory, where one may obtain large-scale pancakes, chains and more or less spherical clusters. The way to describe this situation is to use the recently developed multifractal formalism (Halsey et al. 1986, see also a poster by V. Martinez at this Symposium). The trick here is to study the scale dependence (dimension) of the sum

$$
\beta=\sum_{p_{i}>0} p_{i} q,
$$

where $p_{i}=N_{i} / N$ is the relative number of galaxies in the $i-$ th box of side 1 , and the exponent $q$ can be chosen freely. If we take $q=0$, this sum counts the number of occupied regions and we get once more the normal fractal dimension. With increasing $q$ the sum (2) is dominated by more dense regions, and negative values of $q$ pick out the most rarefied regions of the sample. For given scale size 1 , one can transform the 
quantities $q$ and $\beta$ into more physical parameters - the exponent $\alpha$, describing a local density law through the mass - scale relation $\mathrm{m}(1) \sim 1^{a}$ and the fractal dimension, $f$, of all regions with a common scaling exponent $a$. The resulting $f(a)$ curve is called a multifractal spectrum and describes well both the local structure and the geometry of the distribution. A few examples are good enough to show this - for a classical self-similar fractal the spectrum reduces to one peak, with $a=f=D$; for a mixture of sheets and chains one gets two peaks in the spectrum at $a=2$ and 1 , and so on.
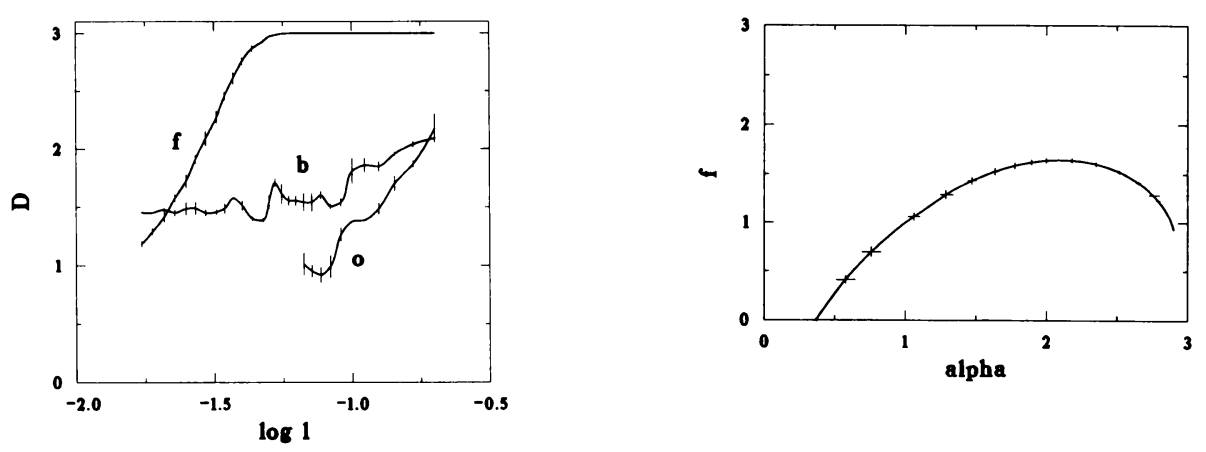

Fig. 4 (left). Fractal dimensions, D, of the observed Coma sample $(o)$, the full ( $f$ ) and biased (b, bias level $n=2$ ) CDM simulations versus scale, 1 (in units of the sample size).

Fig. 5 (right). Multifractal spectrum - fractal dimension, $f$, versus scaling exponent, $a$, for a biased axion simulation (case ' $b$ ' in Fig. 4). The scale $1=1 / 20$-th of the sample size.

Thus, even in the case of a constant fractal dimension it is better to find the multifractal spectrum to reveal the real fractal content of the data. Our example, displayed for the biased model case in Fig. 4, is presented in Fig. 5. The spectrum starts at $a \approx 0.4$, corresponding to the most concentrated region of the model, and ends near $\alpha \approx 2.8$ (the most rarefied regions have approximately uniform density, $\left.m(r) \sim r^{3}\right)$. The fractal dimension of those regions $(f \approx 1)$ is not much less than the full fractal dimension of the sample $D=f_{\max } \approx 1.5$. We see that the spread of local density law (a) and associated fractal dimension ( $f$ ) is rather large and we have a clear case of fractal mixture.

The multifractal approach is evidently useful for comparing simulated models and observations, testing the features of density distributions that could not be tested before. Perhaps even more important is the possibility of revealing distinct features in the spectra of the observed structure which will reflect distinct physical mechanisms influencing the formation of the structure. In this context we may think that the low density regions are spherical voids while the higher density regions are filamentary in nature. This could be quantitatively tested, revealing itself in the $f(\alpha)$ curve. 


\section{CONCLUSIONS}

We have shown that:

(i) giant and dwarf galaxies occupy practically identical regions in space, in conflict with a conventional CDM galaxy formation scenario;

(ii) unbiased model samples are in conflict with observations, demonstrating the need for a biased galaxy formation mechanism;

(iii) the dimension of the observed galaxy distribution does not remain constant for any large scale interval, casting doubt on simple fractal models.

We thank Dr John Huchra for supplying us with a copy of his redshift compilation (Huchra 1983) and our collaborator Mirt Gramann for permission to use her results prior to publication.

\section{REFERENCES}

Dekel, A. and Silk, J., 1986. Astrophys. J. 303, 39.

Doroshkevich, A.G., Shandarin, S.F. and Saar, E., 1978. Mon. Not. R. astr. Soc. 184, 643.

Einasto, J., Einasto, M., Gramann, M., Melott, A. and Saar, E. 1986a. Tartu Astr. Obs. Preprint A-7.

Einasto, J., Gramann, M., Einasto, M., Melott, A., Saar, E. and Saar, V. 1986b. Tartu Astr. Obs. Preprint A-9.

Einasto, J., Joeveer, M. and Saar, E., 1979. Tartu Astr. Obs. Preprint (Mon. Not. R. astr. Soc. 193, 353, 1980).

Einasto, J., Klypin, A. A. and Saar, E., 1986. Mon. Not. R. astr. Soc. 219, 457.

Einasto, J., Klypin, A. A., Saar, E. and Shandarin, S.F., 1984. Mon. Not. R. astr. Soc. 206, 529.

Einasto, M., 1987. Preprint.

Fisher, J.R. and Tully. R.B., 1981. Astrophys. J. Suppl. 47, 139.

Gott, J.R., Melott, A.L. and Dickinson, M., 1986. Astrophys. J. 306, 341.

Gramann, M., 1987. Preprint.

Halsey, T.C., Jensen, M.H., Kadanoff, L.P., Procaccia, I. and Shraiman, B.I., 1986. Phys. Rev. A 33, 1141.

Hockney, R. W. and Eastwood, J. W., 1981. Computer Simulation Using Particles, McGraw Hill, N. Y.

Huchra, J. P., 1983. Redshift compilation.

Jones, B.J.T., Martinez, V., Saar, E. and Einasto, J., 1987. Preprint. Kaiser, N., 1984. Astroph. J. 284, L9.

Lachieze-Rey, M., 1986. Preprint.

Mandelbrot, B.B., 1982. The Fractal Geometry of Nature. Freeman and Co., San Francisco.

Saar, E., Einasto, J., Einasto, M. and Gramann, M., 1987. Preprint. White, S.D.M., Frenk, C.S., Davis, M. and Efstathiou, G., 1986. Preprint Steward Obs. No. 620.

Zeldovich, Ya.B., Einasto, J. and Shandarin, S.F., 1982. Nature, $300,407$. 